Article

\title{
Bottom-Up Electrification Introducing New Smart Grids Architecture-Concept Based on Feasibility Studies Conducted in Rwanda
}

\author{
Bartosz Soltowski *, David Campos-Gaona ${ }^{\mathbb{D}}$, Scott Strachan and Olimpo Anaya-Lara \\ Department of Electronic and Electrical Engineering, University of Strathclyde, Glasgow G1 1XQ, UK; \\ d.campos-gaona@strath.ac.uk (D.C.-G.); scott.strachan@strath.ac.uk (S.S.); \\ olimpo.anaya-lara@strath.ac.uk (O.A.-L.) \\ * Correspondence: bartosz.soltowski@strath.ac.uk
}

Received: 30 April 2019; Accepted: 14 June 2019; Published: 25 June 2019

\begin{abstract}
Over the past eight years, off-grid systems, in the form of stand-alone solar home systems (SHSs), have proved the most popular and immediate solution for increasing energy access in rural areas across the Global South. Although deployed in significant numbers, issues remain with the cost, reliability, utilization, sustainability and scalability of these off-grid systems to provide higher-tiered energy access. Interconnection of existing stand-alone solar home systems (SHSs) can form a microgrid of interconnected prosumers (i.e., households owning SHS capable of producing and consuming power) and consumers (i.e., households without an SHS, and only capable of consuming power). This paper focuses on the role of a smart energy management (SEM) platform in the interconnection of off-grid systems and making bottom-up electrification scalable, and how it can improve the overall sustainability, efficiency and flexibility of off-grid technology. An interconnected SHS microgrid has the potential to unlock latent generation and storage capacity, and so effectively promote connected customers to higher tiers of energy access. This approach can therefore extend the range of products currently used by people located in the remote areas of developing countries to include higher-power devices such as refrigerators, TVs and potentially, electric cookers. This paper shows the results of field studies in the Northern Province of Rwanda within off-grid villages where people mainly rely on SHSs as a source of electricity. These field studies have informed further simulation-based studies that define the principal requirements for the operation of a smart energy management platform for the interconnection of SHSs to form a community microgrid.
\end{abstract}

Keywords: bottom-up electrification; interconnected solar home systems; microgrids; smart energy networks

\section{Introduction}

There are 1.1 billion people living without access to electricity in the world. Former UN Secretary General Ban Ki-moon referred to energy as "the golden thread that connects economic growth, social equity and environmental sustainability", offering an opportunity for improved living standards for some of the world's poorest people living in remote rural locations across the Global South. The UN's Sustainable Development Goal No.7 represents a call to action on the part of the international community to "ensure access to affordable, reliable, sustainable and modern energy for all" by 2030 [1].

Most of those living without electricity reside in remote rural areas of developing countries with no prospect of connecting to the national power network. This is due to the large costs associated with grid extension (around $\$ 1000$ per household connection in Rwanda [2]), which means this is not economically viable for the rural electrification of base-of-the-pyramid communities. As a result, 
connection fees often must be fully subsidized by the local government in order to provide electricity for everyone in the country by 2023 (electrification target in Rwanda) [3].

To date, off-grid renewable-based systems have presented the most viable means of achieving "energy for all" [4], providing access to 360 million people around the globe [5]. As a result, for the last eight years, people previously relying on kerosene lamps for lighting have been given a chance to use stand-alone systems (not connected to the national network) [6] and pay for them on a monthly basis, instead of covering significant upfront costs. This business model is preferred by those with low financial capacity to invest in a new off-grid connection [7].

Off-grid solutions typically generate electricity for a local community (microgrids) or for a single household. A solar home system (SHS) is typically comprised of a PV module, 12V (typically) energy storage, charge controller and several loads, as seen in the Figure 1.

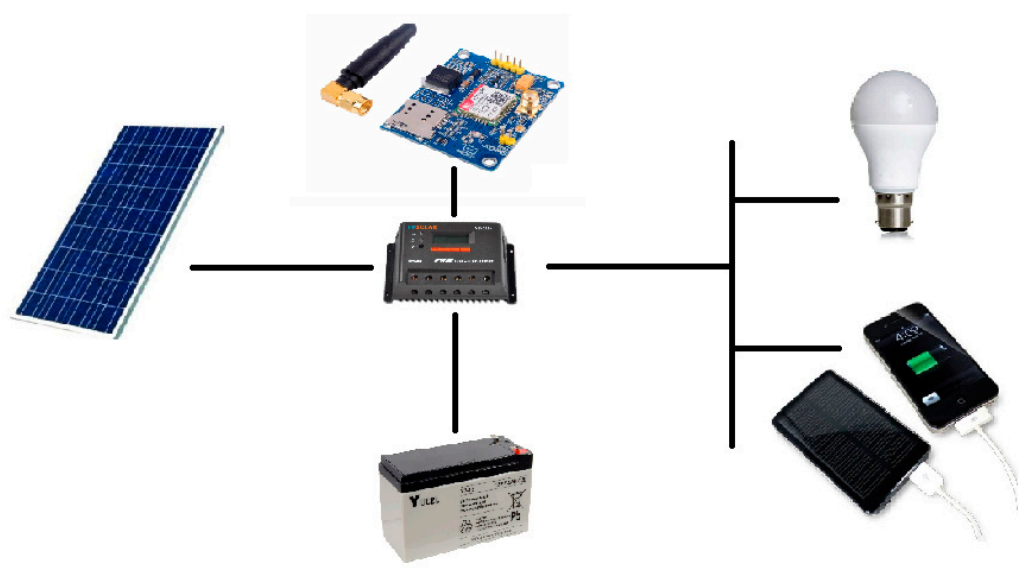

Figure 1. Typical architecture of a solar home system (SHS).

These off-grid solutions generally succeed in providing base-of-the-pyramid communities with a Tier 1 level of energy access, based on ESMAP's (Energy Network Management Assistance Program) multi-tier framework for measuring energy access. They can have a significant effect on the health and wellbeing of those living off-grid, as they reduce household air pollution arising from kerosene lighting, and introduce benefits for education (lighting classrooms for evening study) and commerce through productive uses of energy (e.g., local shops, phone charging, etc.) [8]. However, technical limitations associated with stand-alone SHSs restrict their use to servicing lower powered devices, and so generally provide only basic access (i.e., Tier 1). The Energy Network Management Assistance Program (ESMAP) [9] has developed a multi-tiered framework for measuring energy access, which defines ascending tiers of energy access (0-7) based on capacity, duration reliability, quality, affordability, legality and health and safety. Tier 0 represents customers with no electricity access, while higher level tiers represent the levels of energy access generally associated with a reliable grid connection. Stand-alone SHSs are generally capable of providing Tier 1 level of electricity access, while microgrids and minigrids are generally capable of delivering Tier $2-3$ levels of access and stimulate local businesses $[10,11]$. Table 1 shows how higher power appliances can be accommodated by higher tiers of energy access.

Table 1. Multi-tier Framework for Energy Access.

\begin{tabular}{cc}
\hline Tier Number & Appliances \\
\hline 1 & Lighting + Phone Charging \\
2 & Telecommunication, Fans \\
3 & Refrigeration, Fans \\
4 & Access to clean water \\
5 & Public water, public water works, sanitation \\
6 & Income generating activities \\
7 & Cooking, air-cooling \\
\hline
\end{tabular}


ESMAP's Multi-tier Framework for Energy Access also specifies the quality of power supply at each stage of electrification progression. For lower tiers, duration of electricity available is strictly limited by inadequate generation or storage capability of the introduced system. Progressing to higher tiers, power availability significantly improves, and electricity becomes more affordable and of better quality. Categories for a quality of power supply within each tier are illustrated in Figure 2.

\begin{tabular}{|c|c|c|c|c|c|c|}
\hline & Tier 0 & Tier 1 & Tier 2 & Tier 3 & Tier 4 & Tier 5 \\
\hline Capacity & $\begin{array}{c}\text { No } \\
\text { electricity }\end{array}$ & $1-50 \mathrm{~W}$ & $50-500 W$ & $500-2000 W$ & \multicolumn{2}{|c|}{$>2000 W$} \\
\hline Duration & $<4 \mathrm{hrs}$ & \multicolumn{2}{|c|}{ 4-8hrs } & 8-16hrs & $16-22 \mathrm{hrs}$ & $>22 \mathrm{hrs}$ \\
\hline Reliability & \multicolumn{4}{|c|}{ Unscheduled outages } & \multicolumn{2}{|c|}{$\begin{array}{c}\text { No unscheduled } \\
\text { outages }\end{array}$} \\
\hline Quality & \multicolumn{3}{|c|}{ Low quality } & \multicolumn{3}{|c|}{ Good quality } \\
\hline Affordability & \multicolumn{2}{|c|}{ Not affordable } & \multicolumn{4}{|c|}{ Affordable } \\
\hline Legality & \multicolumn{3}{|c|}{ Not legal } & \multicolumn{3}{|c|}{ Legal } \\
\hline Health \& Safety & \multicolumn{3}{|c|}{ Not convenient } & & \multicolumn{2}{|c|}{ Convenient } \\
\hline
\end{tabular}

Figure 2. Quality of Power Supply for different Energy Access Tiers-provided by ESMAP.

\subsection{Limitations and Benefits of a Bottom-Up Electrification Approach}

While off-grid solutions have proven to be an effective and agile means of delivering basic (Tier 1) energy access, it can be difficult to reinforce and upgrade generation and storage capacities associated with these systems to accommodate the growth in load demand. As a consequence, communities can become effectively "locked into" these lower tiers of basic energy access.

The quality of service provided by existing off-grid systems, as well as future demand for electricity, was ascertained through field studies conducted between March and April 2018 in a representative village in Rwanda; the findings are presented in this paper. These studies were supported by BBOXX, one of the largest SHS providers in the world. A deeper understanding of the off-grid electrification systems, as well as feedback from those currently relying on installations isolated from the national network, leads to the conclusion that rural regions of the Developing World have a great, untapped potential for a new smart grid concepts, which is presented in this paper. It is primarily based on villages currently with basic access to electricity provided by individual SHSs.

While the concept of interconnected SHSs microgrids is not entirely novel [12-15], available analyses do not present how on-site studies and interviews with potential end-users influence the technology's design.

Furthermore, this paper shows, through simulation and analysis, how these SHS's microgrids can improve the reliability and security of supply, reduce the system cost per head and ultimately the levelized cost of energy supplied. In addition, an SHS microgrid with a suitable SEM platform can open up a peer-to-peer energy trading platform for prosumers to generate income by exporting surplus energy, and consumers to access energy by simply connecting to this network and without the need to invest in a SHS.

Before introducing this new concept for electrification, results from field studies in Rwanda are presented. They were undertaken in the Northern Province of the country, where densities of BBOXX installations are the highest. This part of the research was particularly important, as it captured the baseline levels of energy access achieved through the provision of stand-alone SHSs, which could then be compared with the level of energy access determined through the simulation of an interconnected SHS microgrid. Therefore, the paper will show, through simulation and analysis (based on real case-study data), how these SHSs microgrids can improve energy access in terms of improved reliability and security of supply, reduced system cost per capita, reduced levelized cost of energy and accommodation of increased load demand (e.g., with higher power appliances). In a 
broader sense, this analysis will offer supporting evidence for the bottom-up electrification paradigm represented in Figure 3.

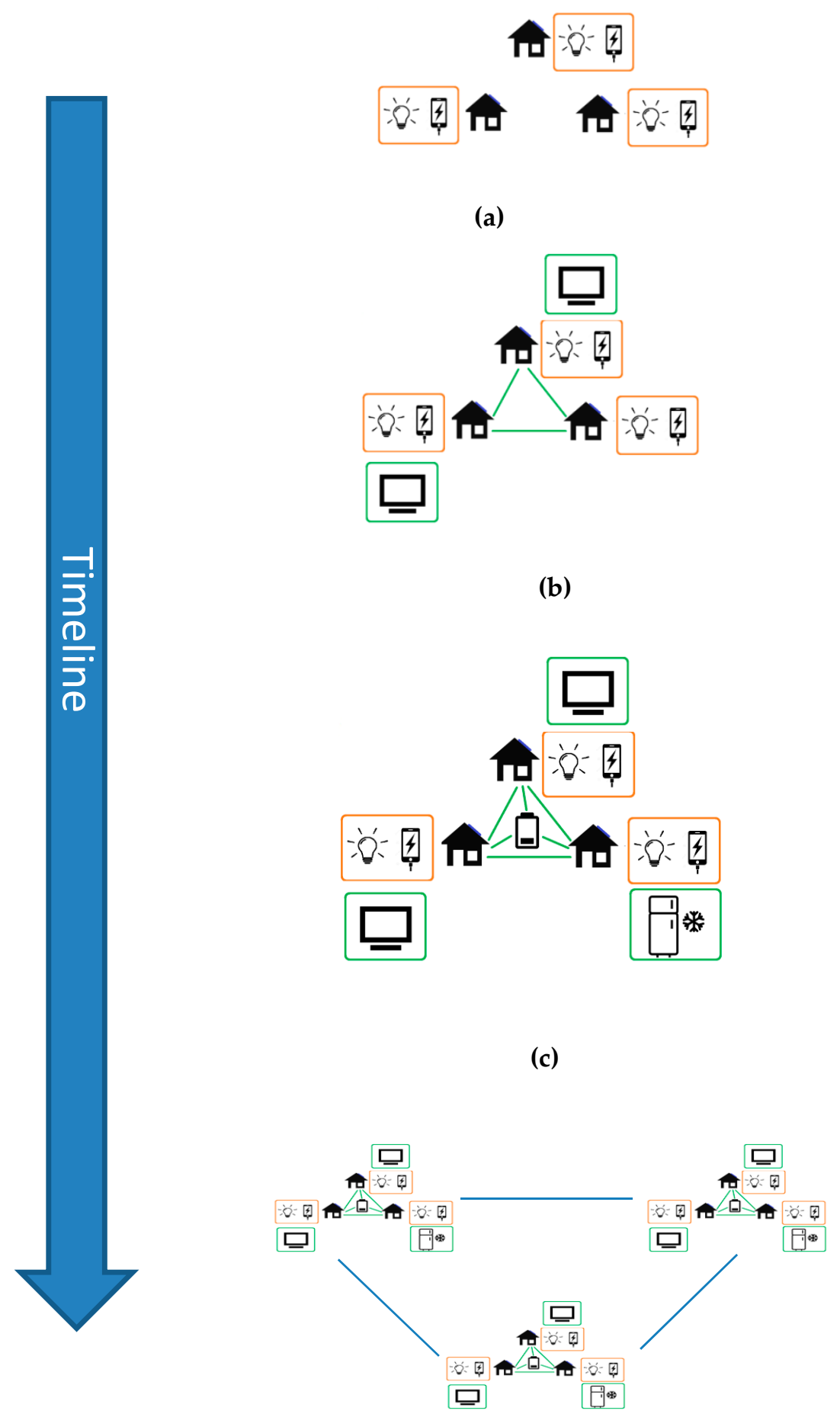

(d)

Figure 3. (a): Tier 1 access to electricity provided by standalone SHSs. (b): Tier 2 access to electricity provided by interconnecting SHSs. (c): Tier 3 access to electricity provided by adding centralized storage within a microgrid of interconnected SHSs. (d): Improved access to currently available appliances by interconnecting microgrids. 


\subsection{Bottom-Up Transition and Scalable Methods to Meet Demand Growth}

Figure $3 \mathrm{~b}$ shows how it is possible interconnect existing SHSs to bring all the benefits of a microgrid (and more) to a community, without the need for additional installed generation or storage capacity. Therefore, at the cost of interconnecting cables and the Smart Energy Management (SEM) platform discussed in this paper, and no additional installed generation or storage capacity, customers can ascend the energy access ladder using the same SHS assets already installed on-the-ground. Figure 3c shows how the addition of some centralized storage and generation could then be incorporated to deliver up to Tier 3 levels of energy access, while Figure 3d shows Tier 4 levels potentially being achieved through interconnection of microgrids.

According to a Global Off-grid Lighting Associating (GOGLA) report from 2018, the rural poor may initially gain energy access through the introduction of pico-PV products (e.g., solar lanterns, mobile phone chargers). Over time, as people become familiar with these basic appliances they may invest in an SHS, which will enable a better, though still basic, level of energy access (lighting and phone charging). Achieving energy access levels beyond basic lighting and phone charging, capable of powering higher-power appliances such as TVs or refrigerators, will require people to often switch to systems with higher capacity, replacing old for new.

At the moment, these kinds of transitions are capital intensive, as they require substantial costs to be covered for new generation and storage, as well as the purchase of new higher power appliances. Interconnecting these existing SHS assets represents a new approach to accommodating higher power appliances and increasing energy access levels without the need for the addition of expensive generation and storage capacity. Instead, this approach utilizes the significant amount of electrical energy that is currently wasted by distributed stand-alone SHSs across a village. This energy effectively substitutes new capacity that would need to be added within the off-grid village, which contributes to the highest portion of the system overall. This concept could reduce investment costs in upgrading existing off-grid assets and could provide smooth and affordable multi-tier transition.

Through simulation and analysis, this paper evidences the step-change in energy access introduced to communities (with pre-existing SHSs), through the bottom-up transition from the configuration shown in Figure 3a to that of Figure 3b. The paper also uses this evidence to define the principal requirements for the operation of a Smart Energy Management platform required to facilitate and enable this transition. Providing an SEM platform introduces a modular component to the interconnection of off-grid systems that is required for scalable, bottom-up electrification that can sustainably, efficiently and flexibly meet the growth in customer demand that inevitably evolves in these rural communities.

\subsection{Success of Bottom-Up Electrification in Bangladesh and Challenges of Deployment in East Africa}

The concept of interconnected SHSs is not entirely new, as it has been introduced in Bangladesh by Solshare, where prosumers are connected using 12V DC distribution systems [16]. However, this approach faces fresh technical challenges for deployment in many Sub-Saharan countries in Africa. This paper discusses these challenges in the context of interconnected SHS microgrid deployment in East Africa. The different challenges posed by Sub-Saharan countries, to that experienced in Bangladesh, are mainly attributable to the difference in the topographies of villages in Bangladesh (a country with one of the world's highest population densities) and those in Rwanda, Kenya or Tanzania, where SHS growth has increased significantly in the last five years. However, SHS penetration and concentration remains significantly higher in Bangladesh than in East African countries, where most SHS "owners" are farmers located in villages with much lower population densities, and so exhibiting greater distances between houses (and even greater distance between houses with SHSs) than is evident in Bangladesh. Consequently, interconnection between these households requires systems with higher distribution voltages and new power converter architectures, as well as smart demand side management to maintain the balance between generation and demand. A new smart network controller needs to understand and bypass existing SHSs infrastructure-BBOXX and other SHS distributors in East Africa offer fully integrated systems with remote monitoring (RM) incorporated. These systems also monitor digital 
payments associated with daily system usage. As a result of these features, the main control hardware embedded within the SHS, designed to manage the RM and digital payments, must remain intact, and not be compromised by the integration of any retrofitted technology (e.g., to enable interconnection of these pre-installed SHSs). However, existing solutions and approaches are unable to achieve this through seamless integration with existing SHS control hardware and functionality, requiring the bypass of the existing SHS control architecture. More specifically, existing SHS control units are replaced by new control units that must also incorporate these RM payments, charge controller and MPPT (Maximum Power Point Tracking) features, which therefore involves unnecessary duplication of existing control hardware and functionality. This inevitably introduces additional and unnecessary costs associated with the introduction and integration of the platform required for SHS interconnection.

The approach to interconnecting SHSs to form village-scale microgrids can offer a reliable, affordable and scalable means of improving access to electricity by remote rural communities. These community-based SHS microgrids can create a pool of energy that can be shared across its connected customers. Those currently with basic Tier 1 access to electricity offered by standalone off-grid SHSs can interconnect and start sharing electricity within this communal energy pool, which offers an opportunity for connected prosumers and consumers to upgrade their energy access level to Tier 2 without necessarily introducing any additional centralized generation and storage. Critically, this allow customers to connect higher power (or higher tier) appliances that are not technically feasible for stand-alone SHSs to supply alone. The next phase of bottom-up electrification could include interconnections between existing villages in order to further upgrade reliability of supply, which could enable Tier 3 to 4 levels of energy access. This suggests new smart energy management algorithms are required to control the interaction between prosumers and consumers connected across a SHS microgrid.

This report presenting potential for a bottom-up electrification in the Developing World is divided into three sections: First, typical systems deployed in rural regions of Sub-Saharan Africa will be characterized; second, field studies conducted within people currently relying on off-grid technology in Rwanda will be presented; and finally, there will be a deeper discussion of a problem and a potential solution.

\section{Solar Home Systems Load Profiles and Consumption Patterns Analysis}

As stated above, the introduction of interconnected SHS microgrids could introduce a number of social benefits associated with improved levels of energy access. Improved energy access is also defined by improved levels of system reliability, which requires a more technically robust electrical distribution system. Interconnecting existing SHSs introduces a number of technical benefits that serve to form a more robust energy infrastructure, and hence an improved quality and service of electric supply to connected customers.

Minimizing battery replacements by introducing smart energy management functions to balance the load over the interconnected SHS microgrid could reduce numbers of battery failures, resulting in cost reduction for new units [17]. Equal power distribution amongst prosumers/consumers can serve to reduce peak discharge rates of the batteries as a result of increased diversity factor [18]. As a result, heat losses are reduced, improving the overall efficiency of the batteries, which results in prolonged battery lifetimes. This is particularly important because of the significant distances that can exist to the nearest maintenance kiosk. In addition, including SEM functionality, which results in longer SHS battery lifetimes, offers clear financial benefits to SHSs distributors, who typically provide three year warranties on their product.

Demand variations depend on the type of activities conducted within the household and differ based on the users' professions, number of household occupants, etc. Figure 4 presents the average demand across one week for a village with seven SHSs located in the Eastern Province of Rwanda. The results were gathered from the BBOXX Smart Solar Remote Monitoring System. 


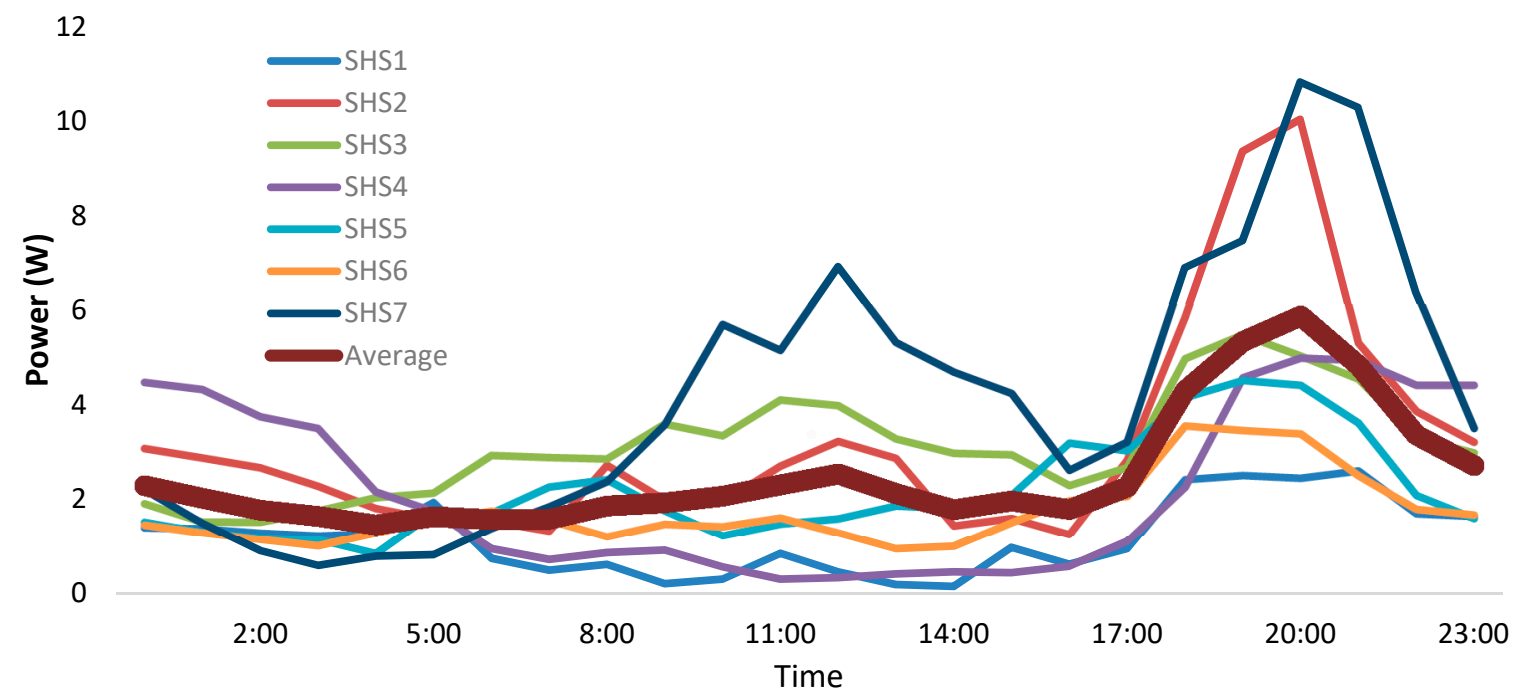

Figure 4. Demand Profiles for BBOXX SHSs.

Consumption profiles of the SHSs analyzed in this document are based on BBOXX SHSs with a PV generation capacity of $50 \mathrm{~W}$. They are equipped with $12 \mathrm{~V}, 17$ Ah sealed lead-acid batteries providing electricity to households after sunset [19]. The basic version of BBOXX SHSs contains two LEDs as well as a phone charger system. Other, more advanced packages provide an extra LED as well as radios or small TVs. The demand specification and power ratings for the most commonly deployed BBOXX SHSs are presented in Table 2.

Table 2. Typical appliances' specifications.

\begin{tabular}{cc}
\hline Device & Rated Power (Watts) \\
\hline LED & 1.1 \\
Phone charger & 1 \\
Radio & 0.75 \\
TV & 8 \\
\hline
\end{tabular}

In order to estimate the amount of electricity which could be shared between users within the interconnected SHSs villages, it was necessary to find a typical energy consumption pattern. This was achieved for a Kageyo village in the Eastern Province of Rwanda due to the high number of BBOXX installation located there (i.e., 64 SHSs in January 2018). Demand profiles over a three month period were analyzed and average daily energy consumption was found for each household. The distribution for the daily energy consumption obtained is illustrated in Figure 5.

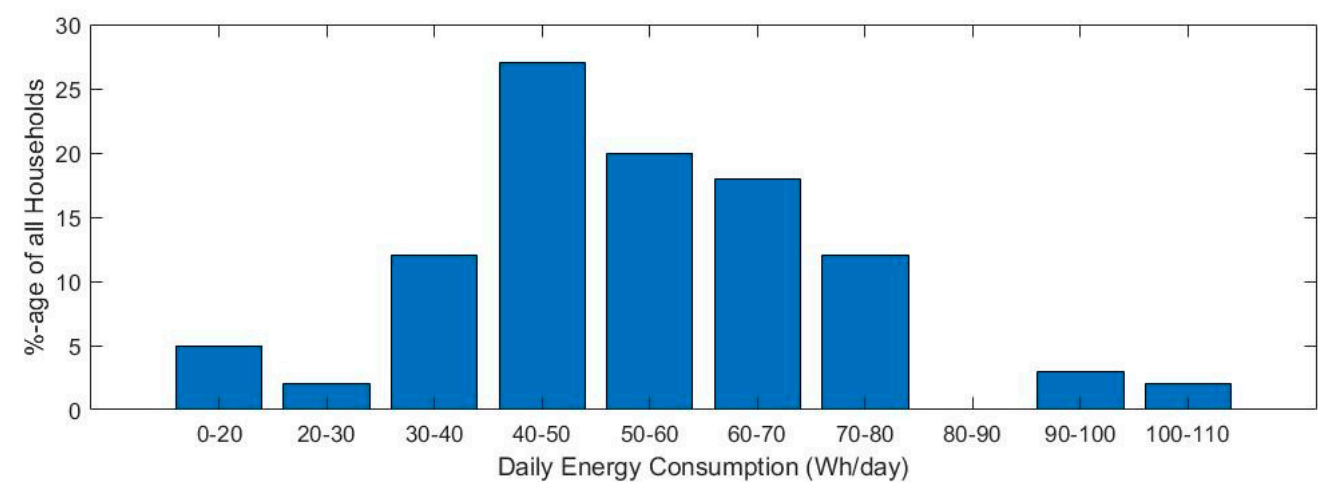

Figure 5. Average daily demand diversity across 64 SHSs. 
The majority of BBOXX customers in the Kageyo village consume between 40 and $80 \mathrm{Wh} /$ day. Further analysis of demand profiles in the village concluded that around $65 \%$ of electricity is consumed when PV generation drops (evening and night). The average energy generated by a single BBOXX unit is estimated at $200 \mathrm{Wh} /$ day, while only $55 \mathrm{Wh}$ of energy on average is consumed, leading to the conclusion that most electricity generation (around 60-65\%) within the village is not utilized and goes to waste.

Currently, stand-alone SHSs with limited storage capacity and limitations on the power consumption associated with the loads that can connect to them, prevent users from accessing this extra generated energy. Unlocking this wasted or surplus energy by offering routes to export to other consumers could therefore enable the connection of new high power appliances across the microgrid. Within a smart interconnected SHS microgrid, load could be shared between two or more systems, allowing energy to be supplied across several prosumers for larger devices.

\section{Off-Grid Market in Rwanda}

This section of the report presents results gathered from a visit in Rwanda where only 23\% of population has access to the national grid at the moment. The high costs associated with main grid extension and reinforcements have led national network operators and the Government Energy Ministry to consider off-grid electrification as a viable solution to the electrification problem. As a result, since 2014, the off-grid energy sector has experienced significant growth [20].

Microgrids and SHSs are widely popular in Rwanda for multiple reasons. Widely accessible communication allows off-grid providers to communicate with assets located in rural regions far from the national grid, which enables remote digital payments as well as remote control of off-grid assets [21]. This enables SHSs operators to upgrade software whenever it is required. Simultaneously, SHS owners can pay on a monthly, weekly or even daily basis for their systems from the most remote regions of the country by using widely popular digital payment platforms.

During four weeks of field studies conducted between March and April 2018, multiple off-grid villages were visited in order to determine the technical requirements for a Smart Energy Management system required for an interconnected SHS microgrid. Several off-grid systems providers, such as BBOXX, Mobisol, MeshPower, Rwanda Energy Group, were interviewed to discuss the proposed interconnected SHS microgrid concept. Furthermore, several villages electrified by stand-alone solutions were visited and users relying on these systems were interviewed.

In the field trial region, the majority of off-grid communities rely on standalone SHSs rather than microgrids for energy access. The total SHS connection cost is typically lower due to the lack of a distribution system. According to MeshPower (the biggest microgrid provider in Rwanda), distribution costs for a typical system providing electricity to 40 households represent up to $60 \%$ of the total microgrid cost [22]. Microgrids are typically deployed in highly populated villages of around 30-40 households within $150 \mathrm{~m}$ from the centralised generation unit. Typical generation capacity ranges from 2 to $4 \mathrm{~kW}$ and is often distributed over $48 \mathrm{~V}$ DC distribution system.

Field studies also proved that in some rural areas of the country, where the national grid could serve customers' demand, due to the poor reliability of the national grid where routine outages are experienced for several hours per day, SHSs were still preferable to some households, due to their higher reliability. It was found that off-grid solutions have been commissioned just several kilometres from the capital city of Rwanda, Kigali, where national grid connection is widely available. However, some families prefer consumption of electricity supplied by the national grid. According to Jean Pascal Niyigena from Rwanda Energy Group, there is a higher status perception associated with a household grid connection in communities that can lead to them being considered as the preferred energy access option over off-grid systems.

Based on field studies conducted in Rwanda, within smaller villages or those with lower population densities, SHSs solutions were the most popular option for electrification. These types of locations were used to conduct studies relating to interconnected SHSs microgrids. 
Topographies of Appropriate Villages Identified for a Bottom-Up Electrification Studies

In order to conduct surveys on which present potential of a bottom-up electrification by integrating SHSs to form a local network with capability to sell and buy electricity between each household, two locations in the Northern Province of the country were selected where BBOXX SHS were prevalent. One of these villages was Ruri in Rulindo District, where around 80 households were located, of which 60 were supplied by SHSs. Typical distances between households ranged from 10 to $80 \mathrm{~m}$, with the majority within 30 to $50 \mathrm{~m}$. These distances are significantly higher than those where microgrid providers such as MeshPower install their systems (between 30 and 40 households within $150 \mathrm{~m}$ from the central point in the village).

Musega village was another village where studies were conducted, where the topography was much different to Ruri village. Musega village was divided into two population centers with around five houses in the first and around 15 in the other. The distance between these two populations centers was around $100 \mathrm{~m}$. Population densities of SHSs were much higher than it was in Ruri village. It was observed that the average distance between households was often very short, ranging from 2 to $10 \mathrm{~m}$ between two neighboring households with BBOXX installations.

Both villages were located in the same region of the country, separated by a distance of $5 \mathrm{~km}$. The map of Rwanda representing popularity of SHSs is illustrated in Figure 6.

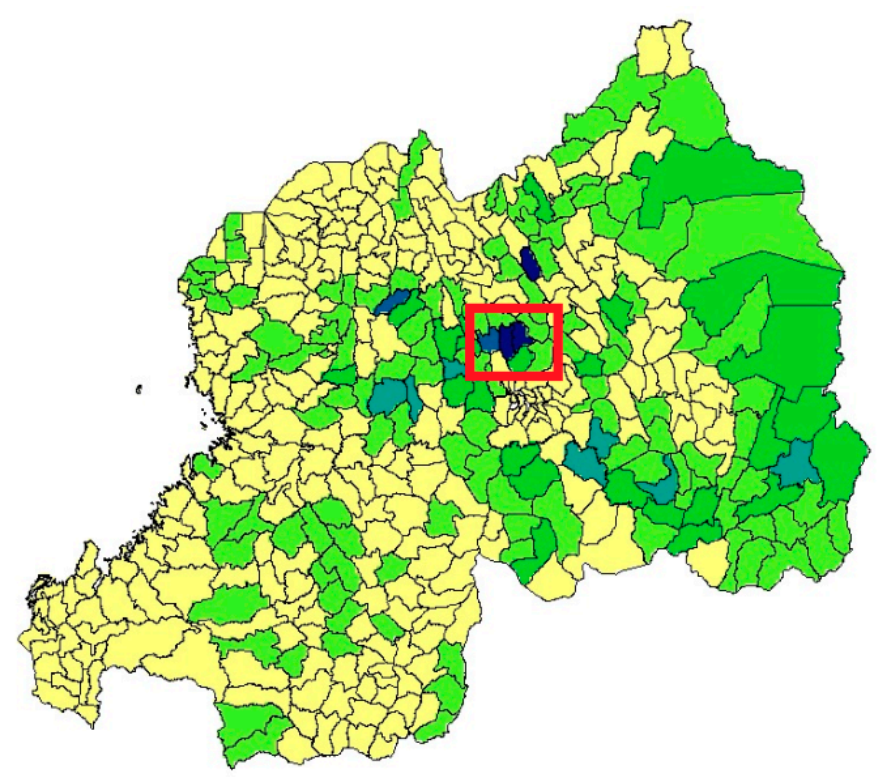

Figure 6. Location of the Case Studies Conducted. Yellow regions represent low rates of SHS penetration, while the green regions represent higher rates of penetration. The dark blue color symbolizes places where SHSs are widely popular. The red square on the map indicates location of the case studies conducted.

In order to determine the quality of services currently offered by SHSs, and the requirements of an SEM platform for interconnecting SHSs in off-grid Rwanda, interviews with customers relying on BBOXX installation were conducted in Ruri village (12 customers) and Musega village (18 customers). Those residing in Ruri village were mainly domestic customers with basic lighting systems and phone chargers, whereas most Musega village BBOXX customers used their installations to run small businesses such as pubs or small shops. The group of BBOXX customers interviewed was dominated by men, with 22 out of 30 responses. Research proved that SHSs technology is equally popular amongst youth (15-25 years old) and elders $(60+$ years old). 


\section{Demand Surveys with Householders Relying on BBOXX SHSs}

All SHS owners interviewed in Ruri and Musega villages have been BBOXX customers for less than 36 months, implying that pay-as-you-go contracts requiring regular payments at least once every month still applied to every user interviewed. At the moment, the only upgrade offered by SHSs distributors is an option to add new appliances (usually small TV, radio, iron or more LEDs) to the system.

\subsection{Feedback from BBOXX SHSs Users}

In general, villagers were satisfied with services offered by BBOXX SHSs, mainly due to significant improvement in light quality over previously used kerosene lamps. After the installation of SHSs, security in the village has also improved as a result of the introduction of external security lights to houses. Some users complained about the lack of capacity offered by the BBOXX SHSs. This may be a result of the low voltage disconnect threshold level, locking the system whenever its capacity is exceeded. In some cases, high amounts of capacity are still available within the battery; however, the system operator does not allow users to access this unless they upgrade their contract and payment plan. As a result, some families do not show willingness to add more appliances to the system, as they are unaware of this extra "locked" capacity that a BBOXX SHS is capable to offer.

For users running local shops, income generation opportunities were increased due to the provision of basic energy access, allowing them to trade in the evening as well host social events with TVs for several hours every day.

\subsection{Measuring Willingness to Pay for Larger Energy Appliances}

The survey questions were also designed to estimate willingness to pay for the addition of larger appliances to the existing system. The majority of those surveyed pointed to refrigeration systems as the most desired high power item. Some householders also had aspirations to use TVs (bigger than those currently offered by BBOXX). To estimate willingness to pay for these appliances, a pay-as-you-go fee was introduced and presented to the interviewed SHSs users. As a result, it was assumed that by upgrading standalone SHSs to an interconnected SHS microgrid, customers would be given a chance to add extra appliances, currently not capable of being supplied by available standalone systems. Estimated costs were divided between two tariffs: PAYGO is valid for the first three years from the purchase of the appliance (this would be charged once every month). The second tariff would apply after the end of the three-year PAYGO contract and would be used to cover costs of energy required to supply appliances as well as cover maintenance costs of assets connected to the microgrid. The estimated tariffs are illustrated in Table 3.

Table 3. Proposed fees for upgrading systems.

\begin{tabular}{ccc}
\hline Appliances & PAYGO Fee per Month for 36 Months & Fee after 36 Month Period \\
\hline 22" TV & RWF $13,450(\$ 14.85)$ & RWF 6500 $(\$ 7.18)$ \\
Fridge & RWF $16,000(\$ 17.65)$ & RWF $10,700(\$ 11.80)$ \\
$22 "$ TV + Fridge & RWF $22,900(\$ 25.30)$ & RWF $14,700(\$ 16.20)$ \\
Fan & RWF $5000(\$ 5.50)$ & RWF $5000(\$ 5.50)$ \\
DVD Player & RWF $500(\$ 0.55)$ & RWF $500(\$ 0.55)$ \\
Sewing Machine & RWF $11,600(\$ 12.80)$ & RWF $11,600(\$ 12.80)$ \\
\hline
\end{tabular}

Out of the available options interviewed were asked to select the most appropriate option. Aspirations to add larger appliances within villagers with SHSs are illustrated in Figure 7. 


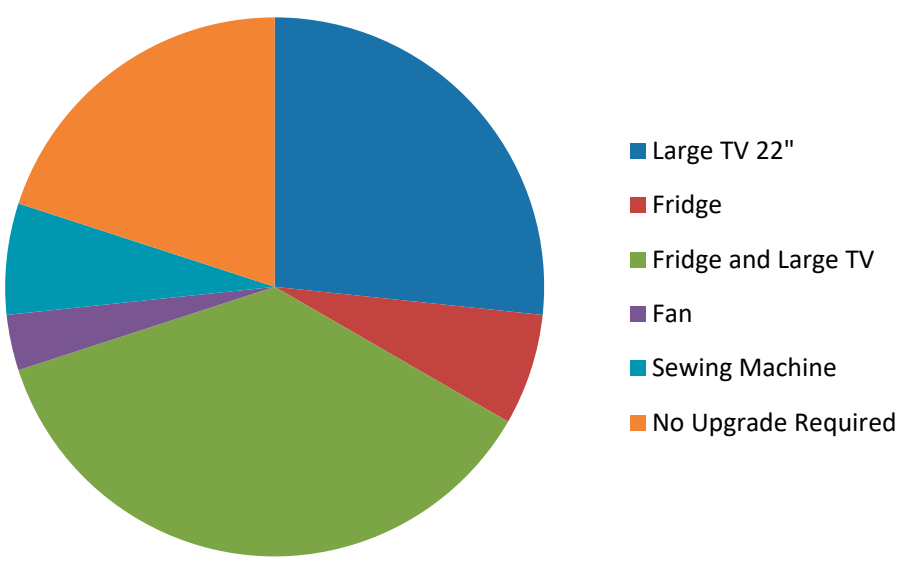

Figure 7. Aspirations to add larger appliances than currently available.

The majority of those living with SHSs show a strong willingness to pay for devices such as fridges or big TVs; therefore, the introduction of smart interconnected SHS microgrids will only make sense if these appliances can be accommodated and supplied. As such, any interconnected SHS microgrid should, at a minimum, be able to provide accommodate $60 \mathrm{~W}$ peak load for a typical small fridge as well as 22 " TV. Other observations associated with the surveyed data presented in Figure 7 are shown below:

- According to Figure 7, willingness to add a sewing machine for income generation activities was not a popular choice, mainly due to lack of elementary knowledge of using these systems.

- Around $60 \%$ of those who showed willingness to add refrigeration showed an interest in using some of its cooling space for rental, believing they could generate extra income by sharing their fridges with other users in the village. As a result, relatively high costs for refrigeration units could be split between two or more households.

- Interestingly, fans as cooling systems were not amongst popular choices. Villagers often asked how the quality of life can be improved by introducing these systems as they were not familiar with fans. According to interviews with BBOXX employees, fans are significantly more popular in regions with higher average temperatures than Rwanda, such as Pakistan, where standard BBOXX systems contain a fan [22].

- A group of BBOXX customers using SHSs upgraded to accommodate a small $8 \mathrm{~W}$ DC TV declared that they would be very interested in upgrading to a new, larger version if it was possible to terminate their previous PAYGO contract for small TVs.

All users interviewed prefer PAYGO for SHS contracts, as in most cases paying full upfront costs for these installations would not be possible. This enables payment of clean electricity tariffs that equates to the cost of energy previously associated with kerosene lamps and phone charging.

Similarly, all BBOXX customers interviewed were keen to participate in the local energy market, to either allow the affordable connection of high power appliances, such as fridges or TVs, or to generate income through the export of electricity to their neighbors.

One common issue that interviewees lacked a clear understanding of was maintenance services provided by SHSs. People do not appreciate the benefits of paying a compulsory maintenance fee after the termination of the 3 year PAYGO contract. This could be a result of a lack of technical knowledge and understanding in relation to the expected battery lifetime, where it will typically lose capacity after 3 or 4 years since the installation (all customers interviewed had their systems for less than 3 years at the time of the interviews). 


\section{Discussion on Field Trial Results and Potential Implications Associated with the Introduction of Interconnected SHSs}

Although feedback provided while interviewing families relying on SHSs in Rwanda was very positive and shows genuine interest in adding new loads to the local demand, there are several implications which need to be considered before introducing the new smart energy networks concept in rural villages of the country. These implications can be categorized as technical, economic and social.

\subsection{Technical Implications}

Technical implications are mainly associated with a new distribution network design as well as with appliances introduced and the need for a SEM system. The majority of SHSs operate as 12V DC systems with appliances tuned for this specific voltage level. Adding new loads to existing infrastructure would either require connection to the existing $12 \mathrm{~V}$ output port provided by an SHS or installation of new power converters providing the appropriate voltage level required for the system. This requires consideration of the availability and suitability of AC and DC electric loads, as this will influence the microgrid architecture.

\subsubsection{AC vs. DC for Microgrid Appliances}

The market for refrigeration systems as well as TVs (the most desired appliances in off-grid Rwanda) is currently dominated by AC appliances, mainly due to the prominence of the AC power grid. Due to economies of scale, AC appliances are significantly cheaper than DC appliances. Despite this fact, it is still worth considering DC as a viable option, mainly due to their high efficiency. It is suggested that DC fridges are more than $20-30 \%$ more efficient than AC [23]. This could be a crucial factor for off-grid progress. DC appliances could introduce more energy savings which result in overall investment cost reduction for generation and storage capacity in the system. DC systems, due to lower peak power consumption, could also prevent networks from being overloaded in the event of very high demand. There is also the prospect of lower customer energy bills due to more efficient consumption. As a result, a more robust cost benefit analysis of AC vs. DC appliances for microgrid level deployment needs to be conducted.

\subsubsection{Appropriate Network Architecture}

A DC microgrid architecture could also bring number of benefits to the distribution network. Synchronization between neighboring DC links is another feature favoring these networks over AC. The introduction of a power inverter could also add fixed losses to the system which are not present while relying on a DC system. Low rates of power for basic appliances, as well as short distances between SHSs, do not make AC a preferable option over DC in the considered environment and applications.

As a result, the reduction in additional hardware required for DC systems, such as power converters, as well as any simplification in system control, would impact positively on the levelized cost of energy, which could ultimately improve the prospects of making higher tiered energy access affordable to poor rural communities.

The issues with DC microgrids arise while considering voltage levels while designing networks due to lack of standards for these kinds of systems except the maximum touch safe $48 \mathrm{~V}$ level, which is used by some off-grid microgrids in Rwanda. Higher voltage levels reduce overall network losses; however, they require more advanced hardware and protection system as they introduce more significant health and safety implications.

\subsubsection{Information and Communication Technology (ICT) Requirements for SHS Microgrids}

Communication aspects are also worth considering when designing smart control for interconnected SHSs microgrids. This is required to capture available generation and demand data. Information shared 
over the network should provide demand side management functions, allowing disconnection of loads whenever the microgrid generation is unable to meet demand. Three types of potential communication systems that may be applicable to these micrigrids are WiFi, LoRa and power line communication (PLC). The pros and cons of each module should be considered with respect to the overall control requirements of the system and the overall power consumption of these systems, due to very limited generation and storage capacities of SHSs.

When designing microgrids based on interconnected SHSs, it is required to introduce plug-and-play systems which do not require major challenges related to system synchronization.

\subsubsection{Demand Side Management (DSM)}

Demand side management (DSM) functions are required to be provided based on overall capacity available in the system. Larger electrical appliances are to be disconnected whenever demand exceeds supply. As a result, suggested architecture for the SEM controller should decouple the existing SHSs' $12 \mathrm{~V}$ output ports from the $12 \mathrm{~V}$ port providing electricity for high power appliances. The proposed architecture of a single controller connected to the $48 \mathrm{~V}$ distribution network.

Based on the discussion above, the proposed controller is equipped with a $12 \mathrm{~V}$ input directly connected to the SHS battery and providing power conversion to share it within the local network. The available capacity of a SHS can also energize local control functions including an on-gate drive circuit, communication systems and remote monitoring containing a micro finance function. The proposed technique for power export could be easily adapted, mainly due to an unregulated $12 \mathrm{~V}$ battery terminal (no power conversion between battery and $12 \mathrm{~V}$ output terminal). As a result, the state of charge of the system could be at any time estimated by the SEM, based on a voltage measurement. This estimation can then be used to send appropriate signals in the microgrid, indicating the capacity available to energize high power devices. Conversely, low voltages on batteries could mean a lack of capacity for supporting fridges which would consequently lead to load shedding.

A $12 \mathrm{~V}$ output terminal is primarily used to provide electricity for devices requiring larger amounts of power, such as fridges and large TVs. The power converter can effectively decouple the $48 \mathrm{~V}$ DC busbar from the $12 \mathrm{~V}$ output terminal. It can provide isolation in the event of low generation capacity or lack of payment for new power appliances.

The full architecture of the proposed microgrid with several SHSs connected to the common $48 \mathrm{~V}$ busbar is illustrated in Figure 8.

\section{$48 \mathrm{~V}$}

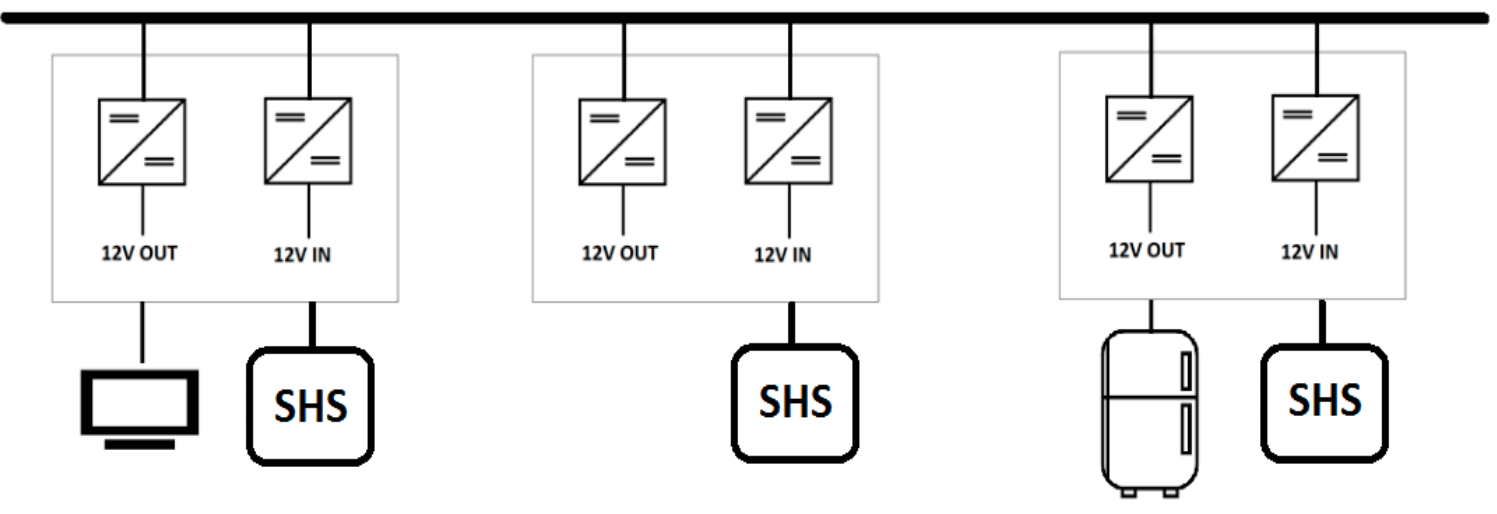

Figure 8. Interconnected SHS microgrid architecture.

Sharing electricity between prosumers using a common $48 \mathrm{~V}$ distribution system could be achieved with a droop control method taking busbar voltage as a reference signal to control power output. One potential issue with this control technique could be introduced by the resistance of the distribution line affecting the perceived reference voltage at each location of the microgrid, thus resulting in variance 
of this reference voltage across these nodes. This is especially the case for two locations separated from each other by a long distance. In such cases, electricity sharing will not be proportional to the aggressiveness of the droop control gain and systems providing voltage support close to the load would tend to provide more power than they were initially meant to. In some cases, this could result in the overcurrent protection being tripped.

These implications could be mitigated by introducing a secondary control reducing the power output of the system exporting electricity and maintaining it at the maximum allowed. As a result, the final power output initially offered by the droop control would not be directly proportional to a voltage drop in the location where the new load was connected. This secondary control would also be required to respond before the overcurrent protection of the SHSs is activated. This would require additional studies on measurements and filter design for each SHS for interconnection.

\subsection{Economic Implications}

The introduction of a bottom-up electrification concept requires deep consideration of the business plan, due to potential risks this technology can bring.

\subsubsection{Investment Uncertainties}

Although results gathered from case studies conducted in Rwanda show a significant willingness to upgrade existing SHSs in order to introduce local energy markets, issues still remain with verifying accurate willingness to pay for larger appliances. Results of the case studies proved a high interest in the introduced concept, but it does not give full confidence of the capabilities to pay for listed appliances. This could be overestimated due to significantly higher costs of ownership for fridges and TVs for the basic SHSs offered so far. Only around 2\% of BBOXX customers fail to pay for SHSs, which would result in systems being returned to the distributor. This implies that further studies are required to precisely estimate the true potential for uptake of additional high power devices in off-grid villages.

\subsubsection{Design of Appropriate Tariffs for Use of the Proposed System}

Designing a fully operational micro transactions scheme and tariffs for those who are willing to add new electrical appliances and those exporting electricity to the network is another challenge. Those with a net energy export need to gain benefits proportional to the rate of energy exported to the microgrid. As a result, electricity should be used only for crucial activities in order to maximize its export. Payments should arise mainly from net energy importers, consuming electricity for larger energy appliances. As a result, with the optimal introduction of new energy markets, all participants should gain benefits, either monetary (those with net energy export) or resulting from new appliances which could lead to new income generation opportunities.

Future demand growth estimation should also be considered while designing accurate tariffs. Smooth adaption of new generation and storage capacity without changing applied tariffs is one of the aspects requiring deeper analysis.

\subsection{Social Implications}

Introducing new equipment such as fridges or TVs and increasing the levels of energy access offered to communities with only Tier 1 access, could also result in new social impacts which are introduced in this section of the paper.

\subsubsection{Development of a New Electrification Plan}

Firstly, it is important to carefully identify the group of villagers that could potentially connect to form an interconnected SHSs microgrid. It is necessary to build a new electrification plan for the whole village, and to define the rate at which demand growth should/could occur, as well as which households can connect first. Providing new appliances for every family showing willingness to use them could 
be catastrophic if there is no evidence of their willingness and ability to pay, mainly due to limited financial capacity within the off-grid environment. Such an occurrence could require installation of extra generation and storage to the system which in the end could be completely impractical due to lack of regular payments completed by some of the users.

One of the solutions is to upgrade SHSs incrementally by first offering interconnections to those who were regular with payments for the initial PAYGO contract. This kind of arrangement could also introduce newly perceived inequalities and feelings of prioritization towards one group of customers over another, leading to a lack of willingness to participate in the local energy market at all and widespread disenfranchisement and disengagement.

\subsubsection{New Appliances Shared between Two or More Households}

There are potentially positive impacts associated with the provision of affordable supplies for larger electrical appliances. By adding a fridge to one of the households, refrigeration space could be shared between two or more families, reducing overall cost per head of using these devices. This kind of adoption of new devices could provide faster and more efficient transition within the access to electricity multi-tier framework, as well as having positive economic and health impacts through the reduction in food waste. Subsistence farmers and agro-businesses can also preserve goods for longer between harvest and consumption or taking to market.

\subsection{Other Potential Barriers}

Other barriers could result from lack of awareness of power ratings of electrical appliances currently used in the off-grid environment. This could be a particularly challenging aspect when connecting larger appliances to the system. Each SHS has its rating indicating maximum power output that could be exported from the battery before internal SHS protection trips. As a result, it is important to equip potential customers with appliances that could be easily energised from the microgrid, while not exceeding maximum ratings of the system. This would suggest that all appliances like fridges or TVs should be fully provided by the network system operator.

\section{Interconnected SHSs Microgrids vs. Investment in Larger SHSs}

Although interconnected SHSs microgrids could introduce new opportunities in the off-grid sector, it is important to show their benefits over other potential solutions, that is, the upgrade of existing standalone SHSs.

While upgrading the individual SHS generation and storage capacity within a village would require significant investments in generation and storage, it would not involve investments in the distribution system. However, one of the major issues associated with this approach is a lack of capacity to utilize all generated energy or that produced by neighboring systems. As a result, standalone systems would normally require more capacity installed per head in order to satisfy the worst-case demand scenario.

This section of the paper presents differences in required capacity for interconnected SHSs microgrids and standalone SHSs capable of supplying Tier 3 level energy access and appliances. The data used to build the comparison are based on a village with 20 SHSs.

Statistics were created in order to present typical demand variations for two scenarios. The first scenario considered standalone systems (with currently available technologies) and assumed that systems can share electricity in the local energy pool. It was found that the average energy consumption per household was $51.69 \mathrm{Wh} /$ day. The average standard deviation for energy consumption for individual SHSs was found to be $12.28 \mathrm{Wh} /$ day.

The second scenario assumed interconnected systems where electricity is equally shared between all prosumers. The average standard deviation of daily energy consumption was found to be $2.36 \mathrm{Wh} /$ day per household. This significant reduction in demand diversity could potentially result in a number of benefits for the overall system. 
A reduction in demand diversity for interconnected SHSs networks could significantly decrease the need to install extra generation and storage to the network to maintain a similar level of reliability of power supply as that presented by standalone systems after upgrading appliances to Tier 3 . This could ultimately result in an overall system expenditure reduction, due to lower investment costs in generation and storage modules, and could provide smoother multi-tier transitions than in the case of incrementally upgrading standalone systems with new capacity.

The next stage of the analysis includes a simulation where systems currently deployed are upgraded by a small 12 V $501 \mathrm{DC}$ fridge, consuming $200 \mathrm{Wh} /$ day, which is a new rate of increase of average system demand per household with an upgraded system. Rate of demand variations for this scenario assume the same ratio of standard deviations over new average energy consumption per day for interconnected and non-interconnected scenarios.

It was estimated that in order to maintain a high level of power system reliability of $95 \%$, the fridge upgrade could be offered for up to 11 of the 20 BBOXX systems, with no need to install additional generation or storage capacity within the interconnected SHSs networks. Under a scenario where 11 out of 20 SHSs are upgraded by new generation and storage capacities with no interconnection, significantly higher levels of cumulative generation capacity are required. The comparison is illustrated in Figure 9.

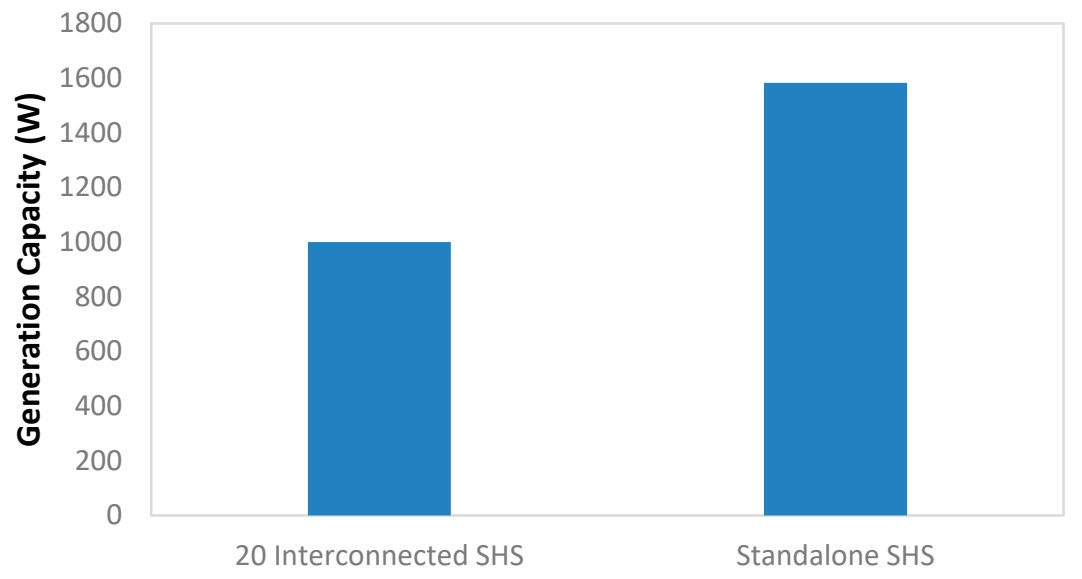

Figure 9. Generation required to supply fridge for 11 BBOXX customers while maintaining the reliability of power supply at $95 \%$.

Upgrading currently deployed SHSs into new with higher generation and storage capacity would require much higher investment costs in PV modules as well as batteries, even though there is still a significant portion of energy available in the village, produced by SHSs without any upgrade, which currently goes to waste.

Providing new standalone SHSs generating enough capacity to energise refrigeration systems would require substantial investment costs for generation and storage capacity as well as for fridge itself. These costs can exceed maximum financial capacities to pay presented by many people in rural regions of Rwanda.

On the other hand, interconnecting existing assets could offer utilisation of already existing generation capacity which currently goes to waste. As a result of this, as well as strong demand diversities presented in people between households, people with existing SHSs installation could make use of that generation capacity to power refrigeration systems for significantly lower costs. New expenditure would be limited to installation of low voltage interconnections between households and refrigeration systems. 
Improved Reliability of Power Supply for Interconnected SHSs Networks over Standalone Systems

One of the challenges while maintaining off-grid assets is their capability to operate without the need for any maintenance, with the exception of routine replacement of balance of system components, most notably the system batteries. Currently deployed SHSs do not present high redundancy and potential failures lead to system deactivations, leaving customers without access to electricity. Within networks of interconnected SHSs, households with power supply issues can still be temporarily supplied by electricity available within a local microgrid, introducing additional sources of electricity than is currently offered by standalone SHSs.

Issues within the network of interconnected SHSs may arise while experiencing potential failures at the distribution level (introduced by a controller itself or distribution line). In order to maximise the reliability of supply in the system, the microgrid should be capable of operating as two or more separated subsystems. This could have an undesirable impact on the part of the network with net energy import. A drop in reliability of supply for the scenario with 20 SHSs, with 11 upgraded to use Tier 3 appliances, is presented in Figure 10.

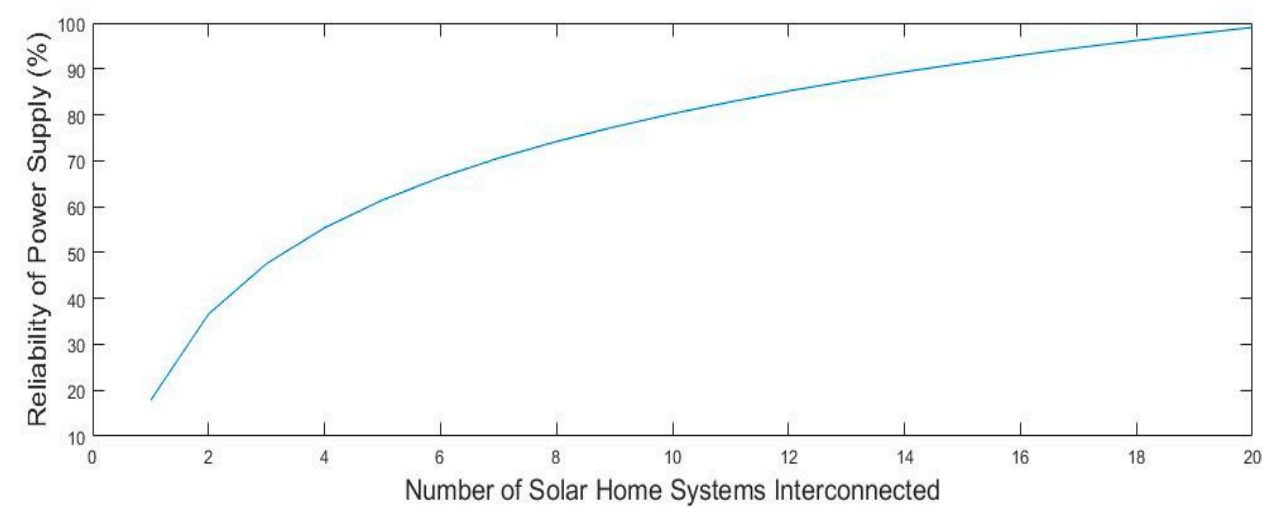

Figure 10. Reliability of power supply and number of prosumers in the microgrid.

Figure 10 proves that losing prosumers in the system due to network failure can drastically reduce the reliability of the power supply. Results illustrated in Figure 10 consider a fixed ratio of users with upgraded SHSs (adding a fridge) over the total number of systems interconnected (ratio of 11:9, as used in the previous steps of this section as the optimal ratio to maintain $95 \%$ energy supply with small DC fridges). The reliability of power supply could vary significantly under scenarios where one isolated network is heavily dominated by systems with fridges. In this case, there might be not enough generation to provide satisfactory power supply.

\section{Conclusions}

In this paper, the concept of bottom-up electrification in rural regions of Sub-Saharan Africa has been introduced, and in particular the transition from Tier 1 to Tier $2 / 3$ that can be enabled through interconnected SHS microgrids. The case for this was evidenced through analysis of data that were gathered and analyzed for communities supported by off-grid solutions offered by BBOXX.

The introduction of this new rural electrification paradigm could vary from country to country. In Rwanda, the most popular SHSs are based on the $50 \mathrm{~W}$ PV (BBOXX) or $100 \mathrm{~W}$ PV (Mobisol) system. Most of these standalone SHSs produce much more electricity than they consume and store (more than $60 \%$ of electricity generated goes to waste). It is proposed that interconnecting these SHSs allows surplus electricity to be more fully utilized, and potentially in the supply of higher power devices, which currently cannot be accommodated using typical standalone SHSs.

Currently, the most desired electrical appliances in off-grid villages in Rwanda are fridges and TVs, declared by $84 \%$ respondents. To date, the only method of adapting these systems to accommodate 
these higher load devices is through the introduction of new generation and storage capacity, requiring significant upfront costs.

Based on analysis provided in this paper, bottom-up electrification introduces significantly smoother and scalable transition for growing demand. It is estimated that interconnected SHSs microgrids required around 35\% less generation capacity than standalone systems in order to keep similar average reliability of power supply of $95 \%$. This is mainly a result of strong demand variation between household, as measured based on installations in Rwanda. As a result, instead of offering new generation and storage technologies (requiring substantial upfront costs) to adapt growing demand, new appliances could be supplied by unused electricity produced by remaining prosumers in the village. Due to widespread communication technologies available in East Africa, it is relatively easy to monitor the microgrid in order to understand when to adapt extra generation/storage to meet growing demand.

Interconnected SHSs microgrids also introduce additional security of power supply in events of losing capability to consume electricity using SHS. Overall reliability of the power supply in the system would remain at high level. This parameter could drastically drop if the microgrid separates into two or more isolated systems, resulting in overall reduction in demand diversity and low reliability of supply.

This approach could be beneficial for many communities with limited access to electricity in Rwanda and neighboring countries where SHSs are widely popular. The situation may look different in other country contexts, where types of off-grid systems are significantly different to those in Rwanda. For example, in Malawi, off-grid solutions currently have a preponderance of pico-solar systems, which have barely enough capacity to power up a single light bulb and a phone charger. As a result, the lack of SHSs on-the-ground there means that interconnected SHS microgrids may yet be a stage of bottom-up electrification not currently accessible. This would imply that in order to provide transition within the multitier framework, interconnection together with new generation and storage capacity would be required in such places. Despite all these difficulties, off-grid sector experiences strong drive from pico-PV modules into bigger SHSs with significantly higher generation capabilities, indicating greater potential for the interconnected SHSs networks in the future.

Following on-site interviews with SHS customers and baseline studies on upgrading standalone SHSs to interconnected SHSs microgrids, further work is required to fully understand all benefits introduced by the concept of bottom-up electrification in the Developing World. At each stage of these studies it is important to consider technical, economic and social aspects in order to precisely match customers' demand with the capability to fully utilize the introduced system.

Author Contributions: Conceptualization: B.S., S.S.; Investigation: B.S.; Methodology: B.S., D.C.-G., S.S.; Technical Analysis: B.S., D.C.-G.; Writing-Review and Editing: B.S., S.S.; Supervision: O.A.-L.

Funding: Project funded by EPSRC_Engineering and Physical Sciences Research Council. Project reference number: EP/L016680/1.

Acknowledgments: Bartosz Soltowski thanks to BBOXX for giving access to remote monitoring data and for on-site support while conducting research in Rwanda.

Conflicts of Interest: The authors declare no conflict of interest.

\section{References}

1. The Secretary-General's High Level Group on Sustainable Energy for All. SE4All, Sustainable Energy for All-A Framework for Action; United Nations: New York, NY, USA, January 2012.

2. Ministry of Infrastructure. Rural Electrification Strategy; Ministry of Infrastructure: Kigali, Rwanda, 2016.

3. Burrough-Boenisch, J. Access to Energy in Rwanda Access to Energy in Rwanda: Impact Evaluation of Activities Supported by the Dutch Promoting Renewable Energy Programme; No. 396; Ministry of Foreign Affairs: The Hague, The Netherlands, 2014.

4. Kabir, E.; Kim, K.H.; Szulejko, J.E. Social impacts of solar home systems in rural areas: A case study in Bangladesh. Energies 2017, 10, 1615. [CrossRef] 
5. International Finance Corporation. 2018 Off-Grid Solar Market Trends Report; International Finance Corporation: Washington, DC, USA, 2018; pp. 1-86.

6. IRENA. Off-Grid Renewable Energy Solutions; No. 2015; IRENA: Abu Dhabi, UAE, 2018; pp. 1-20.

7. Grimm, M.; Lenz, L.; Peters, J.; Sievert, M. Demand for Off-Grid Solar Electricity_Experimental Evidence from Rwanda; SSRN: Rochester, NY, USA, 2017.

8. Lecoque, D.; Wiemann, M. The Productive Use of Renewable Energy in Africa; European Union Energy Initiative Partnership Dialogue Facility: Eschborn, Germany, 2015; pp. 1-9.

9. Energy Sector Management Assistance Program (ESMAP). A New Multi-Tier Approach to Measuring Energy Access Agenda; World Bank: Washington, DC, USA, 2014.

10. Bahaj, A.; Blunden, L.; Kanani, C.; James, P.; Kiva, I.; Matthews, Z.; Price, H.; Essendi, H.; Falkingham, J.; George, G. The Impact of an Electrical Mini-grid on the Development of a Rural Community in Kenya. Energies 2019, 12, 778. [CrossRef]

11. Kirubi, C.; Jacobson, A.; Kammen, D.M.; Mills, A. Community-Based Electric Micro-Grids Can Contribute to Rural Development: Evidence from Kenya. World Dev. 2009, 37, 1208-1221. [CrossRef]

12. BIF. A Survey of the Pico Solar Product Grey Market in Malawi; Business Innovation Facility: Blantyre, Malawi, 2014.

13. Bowes, J.; Booth, C.; Strachan, S. System interconnection as a path to bottom up electrification. In Proceedings of the 2017 52nd International Universities Power Engineering Conference (UPEC), Heraklion, Greece, 28-31 August 2017; pp. 1-5.

14. Hollberg, P. Swarm Grids-Innovation in Rural Electrification. Master's Thesis, KTH School of Industrial Engineering and Management, Stockholm, Sweden, 2015.

15. Hoffmann, M.M.; Ansari, D. Simulating the potential of swarm grids for pre-electrified communities-A case study from Yemen. Renew. Sustain. Energy Rev. 2019, 108, 289-302. [CrossRef]

16. Transition, T.M.E. SOLshare Bottom Up Micro-Grids; The Intersolar Award: Dhaka, Bangladesh, 2016.

17. Narayan, N.; Papakosta, T.; Vega-Garita, V.; Qin, Z.; Popovic-Gerber, J.; Bauer, P.; Zeman, M. Estimating battery lifetimes in Solar Home System design using a practical modelling methodology. Appl. Energy 2018, 228, 1629-1639. [CrossRef]

18. Soltowski, B.; Bowes, J.; Strachan, S.; Anaya-Lara, O.L. A Simulation-Based Evaluation of the Benefits and Barriers to Interconnected Solar Home Systems in East Africa. 2018 IEEE PES/IAS Power Afr. 2018, 1, 491-496.

19. BBOXX BB17 Kit Datasheet; BBOXX Ltd.: Chiswick, London, UK, 2012.

20. International Energy Agency. Energy Access. 2017. Available online: https://www.iea.org/sdg/electricity/ (accessed on 29 April 2019).

21. Bisaga, I.; Puźniak-Holford, N.; Grealish, A.; Baker-Brian, C.; Parikh, P. Scalable off-grid energy services enabled by IoT: A case study of BBOXX SMART Solar. Energy Policy 2017, 109, 199-207. [CrossRef]

22. Zimmerle, D.; Manning, D.T. Optimizing rural village microgrids to provide affordable and reliable renewable electricity in developing countries. In Proceedings of the 2017 IEEE Global Humanitarian Technology Conference (GHTC), San Jose, CA, USA, 19-22 October 2017; pp. 1-6.

23. Opoku, R.; Anane, S.; Edwin, I.A.; Adaramola, M.S.; Seidu, R. Comparative techno-economic assessment of a converted DC refrigerator and a conventional AC refrigerator both powered by solar PV Évaluation comparative technico-économique d' un réfrigérateur converti à courant continu (DC) et d' un réfrigérateur con. Int. J. Refrig. 2016, 72, 1-11. [CrossRef]

(C) 2019 by the authors. Licensee MDPI, Basel, Switzerland. This article is an open access article distributed under the terms and conditions of the Creative Commons Attribution (CC BY) license (http://creativecommons.org/licenses/by/4.0/). 